\title{
A sharp contraceptive patch
}

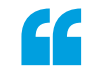

\section{Using our}

patch, a

woman

could self-

administer a

contraceptive

hormone

by briefly applying the patch to her skin only once per month

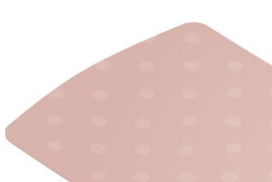

Long-term hormonal contraception is costly and usually requires frequent dosing or medical procedures.

Long-acting, low-cost contraception by self-administration would greatly benefit women, in particular, in regions with limited access to health-care providers. Now, writing in Nature Biomedical Engineering, Mark Prausnitz and colleagues report a bubble-microneedle patch for the delivery and sustained release of a contraceptive hormone, providing painless, cheap and easy-to-use contraception.

The researchers fabricated the patch by moulding polymer microneedles made of biodegradable polylactic acid (PLA) and polylacticco-glycolic acid (PLGA) - both biomaterials used in US Food and Drug Administration (FDA)approved products - together with the contraceptive hormone levonorgestrel incorporated within the polymer matrix. The hormone can be released by diffusion or polymer degradation over the course of weeks or months. "The polymer microneedles have been designed to have the appropriate mechanical strength, biocompatibility and manufacturing processability to be applied as a patch, and they can encapsulate a contraceptive hormone in their matrix," says Prausnitz.

The microneedle patch is applied to skin by manually applying pressure for 5 seconds, causing rapid detachment of the microneedles from the patch. "A key innovation in this study is the mechanism by which the microneedles separate from the patch backing," explains Prausnitz. "We incorporated a small air bubble between each microneedle and the patch backing, which makes the microneedle strong enough to resist compression during insertion into skin and weak enough to be easily sheared off after insertion."

The researchers tested the patch on porcine skin ex vivo, demonstrating that more than $95 \%$ of microneedles detach by manual pressing and that the needles remain embedded in the skin after separation. To assess performance

of the microneedles in vivo, Prausnitz and colleagues applied a patch with a $10 \times 10$ array of microneedles loaded with levonorgestrel to the skin of rats. The hormone is slowly and continuously released from the needles, with a peak plasma concentration $\sim 6$ days after treatment, followed by a slow decrease. Importantly, concentrations of the hormone remained above the therapeutically relevant level for 30 days. "Using our patch, a woman could self-administer a contraceptive hormone by briefly applying the patch to her skin only once per month," comments Prausnitz.

The researchers are now working on the fabrication of larger patches to deliver a dose suitable for human contraception and to provide longer-term contraception (up to 6 months), with the aim to move into clinical trials. In addition, the researchers studied the delivery of vaccines using microneedles made from rapidly water-soluble materials, such as sucrose or polyvinyl alcohol, to encapsulate a vaccine. "We carried out a phase 1 clinical trial of influenza vaccination using microneedle patches, and found that vaccination was well tolerated and at least as immunogenic as conventional intramuscular injection," comments Prausnitz.

Christine-Maria Horejs

ORIGINAL ARTICLE Li, W. et al. Rapidly separable microneedle patch for the sustained release of a contraceptive. Nat. Biomed. Eng. https://doi.org/10.1038/s41551-018-0337-4 (2018) 